\title{
The Englishness of the
}

\section{Museum Britannicum}

Aus heutiger Sicht ist das Museum Britannicum ein höchst sonderbares Unterfangen. ${ }^{1}$ Stutzig machen sollte, daß der gesamte englische Hochadel das Werk subskribiert hat, Wissenschaftler und Künstler haben sich entschieden zurückgehalten. Von Künstlerseite findet sich allein Benjamin West, zwar schon Akademiemitglied, aber noch lange nicht Akademiepräsident, dafür aber bevorzugt von Georg III. als Historienmaler beschäftigt - das mag eine bewußte Anknüpfung an die Reihe der Dukes und Earls erklären, zumal das Werk dem Prinzen von Wales gewidmet ist. Die Erstausgabe erschien 1778, die von Peter Boyle korrigierte und herausgegebene zweite Ausgabe 1791. Das Nachwort merkt an, daß der Zeitpunkt des Erscheinens der Erstausgabe ungünstig sei: mitten im amerikanischen Freiheitskrieg, in dem sich die Kolonien vom Mutterland lossagten - ein Grund mehr für den gebürtigen Amerikaner West, ein Bekenntnis zu England abzulegen. Die Autoren des Museum, John und Andrew van Rymsdyck, die vor allem auch die Zeichner der auf dreißig Tafeln dargestellten Objekte sind, haben die potentiellen Adressaten befragt, was sie in dem Werk dargestellt sehen wollten: jeder etwas anderes. Darum hätten sie eine Mischung aus „Antiquities“ und „Natural Curiosities“ wiedergegeben, nach drei Kriterien: ein paar feine Dinge, einige eher mittelmäßige und ,a few perhaps quite indifferent". ${ }^{2}$

1 John und Andrew van Rymsdyck: Museum Britannicum. Being an Exhibition of a Great Variety of Antiquities and Natural Curiosities. Belonging to That Noble and Magnificent Cabinet, the British Museum. Illustrated with Curious Prints, Engraved after the Original Designs, from Nature, Other Objects; and with Distinct Explanations of Each Figure, London 1778; John und Andrew van Rymsdyck: Museum Britannicum; or a Display in ThirtyTwo Plates, in Antiquities and Natural Curiosities, in That Noble Cabinet, the British Museum, hrsg. v. P. Boyle, London ${ }^{2} 1791$. Das Museum findet in der Literatur nur selten Erwähnung, beinah zu erwartende Ausnahme: Barbara M. Stafford: Kunstvolle Wissenschaft. Aufklärung, Unterhaltung und der Niedergang der visuellen Bildung, Amsterdam/Dresden 1998 (zuerst engl. 1994), 290-303.

2 Rymsdyck 1791 (wie Anm. 1), IV. 
Das ist nur zu wahr, das Werk verzichtet offenbar willentlich auf jede irgendwie nachvollziehbare Ordnung, es gibt noch nicht einmal Gegenstandsabteilungen, sondern nur einen bunten Strauß. Das Museum hat Folioformat, die Abbildungen haben immerhin eine Plattengröße von etwa $33 \times 23 \mathrm{~cm}$. Bei größeren Gegenständen finden sich zwei, bei kleineren mehrere auf einer Seite, sie können, müssen aber nicht unbedingt etwas miteinander zu tun haben. Auf Tafel I haben wir oben ein aus einem Pflanzenblatt gebautes bengalisches Vogelnest und unten ein Wespennest aus Spanisch-Westindien, auf Tafel II oben einen Oculus Mundi genannten Stein aus China, der, in Wasser getaucht, durchsichtig wird, unten eine Muschel mit Perle. Gefolgt auf Tafel III von einem verkrusteten Schwert und einem gleichermaßen verkrusteten Totenkopf, beide im Tiber in Rom gefunden. Der Kommentar macht sich Gedanken über verschiedene Formen der Versteinerung, im Wasser, im Sand etc. Tafel IV bringt ein unbekanntes metallenes, wohl antikes Objekt, die „Antiquaries“ sollen sich darüber den Kopf zerbrechen, gekoppelt ist es mit römischen Insignien. Und so geht es weiter: eine Tafel liefert Eier, beginnend mit einem auffällig blauen Vogelei bis hin zu Krokodilseiern, ein verrottetes Hufeisen aus einer ungarischen Kupfermine scheint nun aus verkupfertem Eisen zu bestehen. Kommentar und Anmerkungen versuchen den Erkenntnisstand zu referieren und zu kommentieren. Kulturgeschichtliches hat sein Vorkommen, etwa wenn eine Muschel abgebildet wird, Pinna marina genannt, was Linné 1748 Pinna Nobilis entspricht, vulgo Steckmuschel. Sie ist gekoppelt mit einem Handschuh (Abb. 1). Das Verbindende besteht darin, daß der Steckmuschel in der Mitte der inneren Muschelschale seidige Fasern wachsen, mit denen sie sich im Sand verankert, man spricht auch vom Bart der Muschel, in der Antike byssus genannt. Dieser byssus ist schon in der Antike in Form von Fransen in Handschuhe eingearbeitet worden. Das Verfahren hat sich, wie die Autoren anführen, in Süditalien bis in die Gegenwart gehalten und auch aus Andalusien lassen sich Beispiele beibringen, wie das abgebildete. Und so geht es weiter, auch mit fragwürdigen Zuschreibungen, etwa wenn ein Stein vom Turm zu Babel vorgeführt wird. Ägyptisches, Römisches, Amulette, Versteinerungen, antike Tränengläser, Korallen in Handform, die Krönung vielleicht ein menschliches Horn, das Mrs. French aus Kent aus dem Hinterkopf gewachsen sein soll, kombiniert mit Kastagnetten „very ancient".

Was im Himmel mag dieses Konglomerat bezwecken? Liest man die Einleitung sorgfältig, besonders die Anmerkungen, so wird deutlich, daß dies eine Werbeschrift ist, die nachdrücklich - auch die Öffnungszeiten werden genannt - den Besuch des Museum Britannicum empfiehlt - jener Sammlung, die den Grundstock des heutigen British Museum bildete, hervorgegangen aus der Stiftung der Sammlung von Sir Hans Sloane 1744 an die Nation, seit 1753 im vom Staat gekauften Montagu House beherbergt, 1756 vermehrt durch die ägyptischen Antiquitäten von Colonel William Lethieullier, 1757 durch die 
The Englishness of the Museum Britannicum

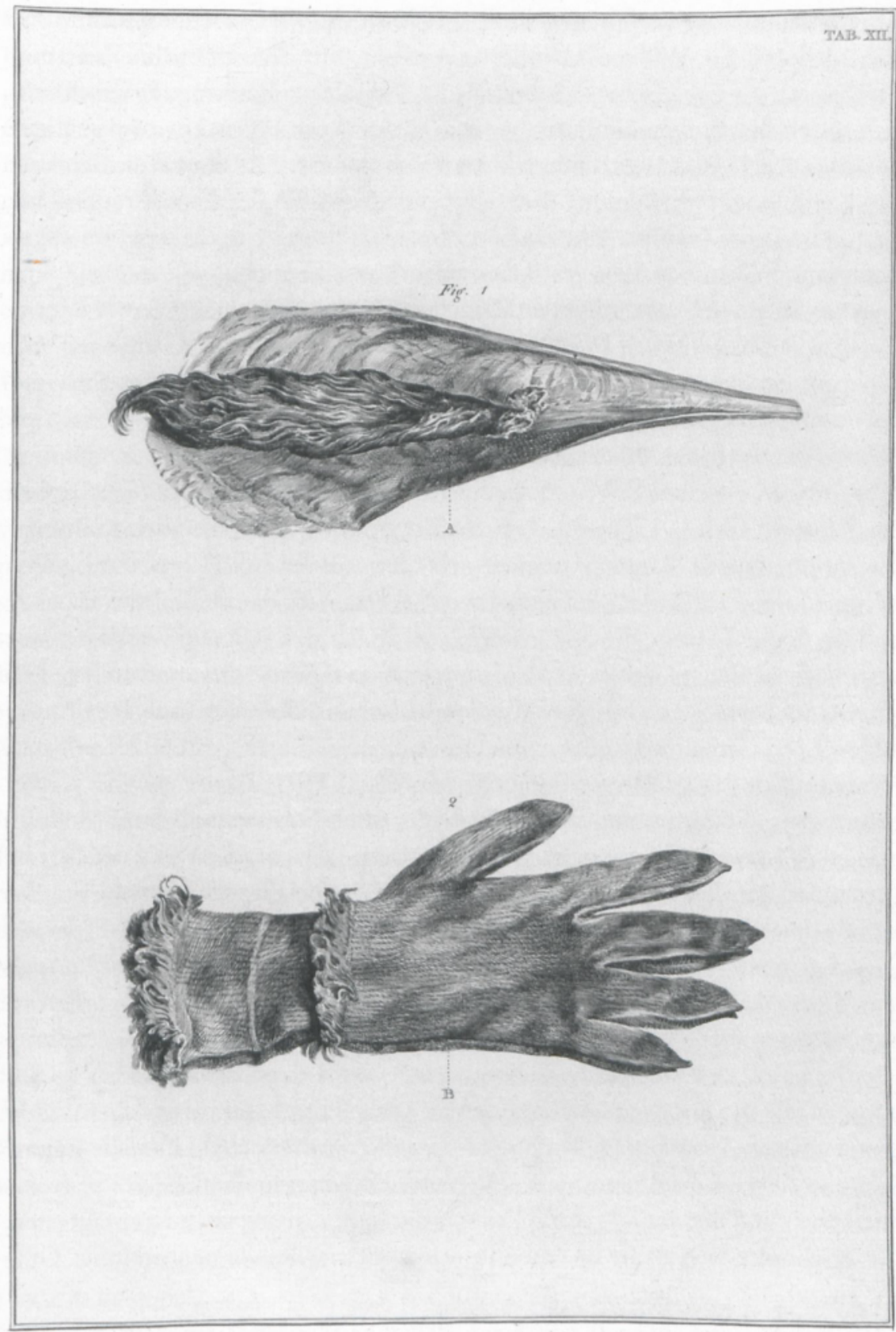

Abb. 1: Museum Britannicum, Tafel XII, Fig. 1 Steckmuschel, Fig. 2 Handschuh 
Antikensammlung von Thomas Hollis, 1772 (nicht 1771, wie die Autoren angeben) durch Sir William Hamiltons Antiken, mit den Vasen im Zentrum, 1802 durch die ägyptischen Altertümer aus Napoleons Raubzug, 1805 schließlich durch die antiken Skulpturen von Charles Townley bis hin zu den Elgin Marbles, die 1816 vom britischen Staat übernommen wurden und die Struktur der Sammlung, vor allem ihr Ausstellungskonzept, ein für allemal radikal änderten und zum Neubau führten. ${ }^{3}$ An Montagu House war zuvor schon angebaut worden, um Townleys Antiken unterbringen zu können.

Die treibende Kraft hinter den Plänen der Errichtung eines Museum Britannicum war die Society of Dilettanti, einst ein Dining Club für Gentlemen mit Operninteresse, die samt und sonders auf der Grand Tour gewesen waren und spätestens seit dem Zeitpunkt der Bildungsreise sammelten. Das Interesse verschob sich, die Society finanzierte Ausgrabungen und systematische Aufnahmen von Antiken und ihre Publikation mit Stuart und Revetts Antiquities of Athens im Zentrum (Bd. 1, 1756; Bd. 2, 1789; Bd. 3, 1794). Ursprünglich wollte die Society ein eigenes Museum bauen. Noch 1785 machte Lord Camelford, selbst Mitglied der Society, dem Sekretär Joseph Banks den Vorschlag, eine Häuserzeile in Oxford Street, die ihm gehörte und an die sich sein eigenes Wohnhaus anschloß, zu einem Club- und Museumsgebäude umbauen zu lassen. Pläne ließ er von Sir John Soane fertigen. Einzige Bedingung dieses großzügigen Angebotes: Lord Camelford wollte einen unmittelbaren Zugang von seiner privaten Wohnung in das geplante öffentliche Museum. ${ }^{4}$ Das ist bezeichnend genug: selbst wenn die Sammlungen der Mitglieder öffentlich zugänglich sein sollten, wie im Museum Britannicum, so war nicht daran gedacht, die Form der Privatsammlung gänzlich aufzugeben. Es sollte ein Sammlermuseum werden, die Bestände der einzelnen Sammlungen sollten beisammen bleiben, selbst die Inszenierungspraxis sollte über weite Strecken beibehalten werden. So setzt auch das British Museum in seiner Frühphase die Tradition der englischen Privatsammlungen fort.

Das hatte einschneidende Konsequenzen. Zum einen haben die Stadtwohnungen wie die Landsitze des englischen Adels Räume mit eindeutigen Funktionen: library, dining-room, drawing-room, gallery, parlour etc. ${ }^{5}$ Über alle Räume breitete sich die Sammlung aus, die zentralen Kommunikationsräume waren die library und der dining-room. Hier war die Inszenierung am ausgeprägtesten, sie erschöpfte sich nicht in Ästhetik - Symmetrie, Pendantanordnung, Grö-

3 Wolfgang Ernst: Historismus im Verzug. Museale Antike(n)rezeption im britischen Neoklassizismus (und jenseits) (Beiträge zur Geschichtskultur Bd. 6), Hagen 1992, 204, 207, $213 \mathrm{ff}$. Der Arbeit von Ernst wird im folgenden einiges verdankt.

4 Ebd., 130.

5 Marc Girouard: Life in the English Country House. A Social and Architectural History, Harmondsworth ${ }^{2} 1980$, bes. Kap. 6-8. 
ßenstaffelung -, sondern folgte nicht selten einer Privatmythologie des Hausherrn. Er, antike Überlieferung hin, antike Überlieferung her, schrieb den Gegenständen ihre Bedeutung zu, versuchte sie durch Kombination zu steigern, entwarf eine private Ikonographie, sammelte gezielt, um den eigenen Kosmos abzurunden, bestellte bei den Agenten in Italien ikonographisch Bestimmtes und sei es, daß es für den Sammler entsprechend zugerichtet wurde. Attribute konnten hinzugefügt werden, ebenso Sockelinschriften, den Figuren wurden neüe Köpfe aufgesetzt, nicht nur Bartolomeo Cavaceppi restaurierte entsprechend. ${ }^{6}$ Die großen englischen Händler, Thomas Jenkins, Gavin Hamilton oder James Byres lieferten Gewünschtes. Sir William Hamilton, der britische Gesandte in Neapel und selbst einer der bedeutendsten Sammler und Ausgräber, konnte ebenfalls als Vermittler dienen.?

Ihren Niederschlag fand die Privatmythologie auf dreierlei Weise: in Sammlungskatalogen, in denen sich antiquarisches Wissen mit gezielter Privatmythologisierung verband, und die Grenzen zwischen historischem Faktum und literarischer Fiktion fließend waren, nicht selten verfaßt von antiquarischen Profis. Ferner durch den Hausherrn selbst, der in der Kommunikation mit gleichgestellten Gästen und Besuchern als Cicerone des eigenen Kosmos fungierte und drittens in Bildern der Sammlung, die nicht etwa bloß das Aussehen der Sammlungsräume dokumentierten, sondern in einem Idealbild der Sammlung den unterlegten Sinn forcierten.

Im Falle von Charles Townley lassen sich die verschiedenen Dimensionen in ihrem Zusammenspiel besonders gut greifen. Townley war ein begnadeter Führer durch seine eigene Sammlung, er galt nicht nur als äußerst kenntnisreich, sondern würzte seine Führungen „by pleasantry and anecdote ${ }^{\text {" }}$, wobei die Anekdoten sich auf Auffindungs- und Erwerbungsumstände bezogen haben dürften, „pleasantry“ dagegen wohl nicht nur kleine Scherze meint, sondern eher einen spielerischen Umgang mit der Bedeutungsdimension der Gegenstände. Immer geht es dem Engländer um deren Verlebendigung, nicht um die Markierung historischer Ferne, sondern um fortlebende Wirksamkeit, und intellektuelle spielerische Umkreisung läßt sie für die Vorstellung agieren. Wenn Townley seine Sammlung von Johann Zoffany im Gemälde darstellen läßt nach dem Vorbild von dessen Tribuna-Bild, in dem im Zentrum der Uffizien

6 Kat. Vases and Volcanoes. Sir William Hamilton and his Collection, hrsg. v. Ian Jenkins/ Kim Sloan, The British Museum, London 1996; Kat. Grand Tour. The Lure of Italy in the Eighteenth Century, hrsg. v. Andrew Wilton/Ilaria Bignamini, Tate Gallery, London 1996 und natürlich: Francis Haskell/Nicholas Penny: Taste and the Antique. The Lure of Classical Sculpture. 1500-1900, New Haven/London ${ }^{2} 1982$.

7 Kat. Vases and Volcanoes (wie Anm. 6); bes. Kat. Grand Tour (wie Anm. 6), 257.

8 Ernst 1992 (wie Anm. 3), 177 (Henry Ellis nach einem Besuch bei Townley), ausführlich zu Townley ebd., 165-200; ferner Kat. Grand Tour (wie Anm. 6), 257-262. 
eine Idealversammlung bedeutender Kunstwerke vorgestellt wird, von englischen Grand Touristen betrachtet und nicht selten individuell auf diese bezogen, so daß eine Lektüre des Bildes auf mehreren Ebenen möglich ist, dann ist die Townleysche Bibliothek ebenfalls Erscheinungsort der wichtigsten Antiken dieses Sammlers, und der Diskurs am Tisch kreist um Townleys Lieblingsskulptur, die sogenannte Iris oder Klythia, die spielerisch als Townleys Braut bezeichnet wurde. ${ }^{9}$ Wortführer im Diskurs ist Pierre-François Hugues d'Hancarville, während Townley gesondert in strengem Profil den um ihn drapierten antiken Büsten zugeordnet ist: auch hier im doppelten Sinn eine Verschränkung von Kunst und Leben.

In Townleys Haus schrieb d'Hancarville sein Hauptwerk Recherches sur l'origine, l'esprit et les progrès des arts de la Grèce, 3 Bände, London 1786 - schon der Titel ist höchst bezeichnend: der Ursprung ist im Mythos der Vorzeit verborgen, sein Geist wirkt bis in die Gegenwart, animiert immer noch die überlieferte antike Skulptur - aber nur, wenn der Sammler diesen Geist wach hält. Townley hatte in den sechziger und siebziger Jahren gleich drei ausgesprochen ausführliche Grand Tours unternommen, eine vierte wurde nur durch den Ausbruch des Krieges mit Frankreich verhindert. Er sah in Rom die Entstehung des Museo Pio-Clementino, an dessen Zustandekommen auch die Society of Dilettanti direkt und indirekt Anteil hat, er pflegte engsten Austausch mit den Antiquaren und Sammlern, kaufte in großem Stil, er forschte selbst intensiv. Für die Anfertigung des Kataloges seiner Sammlung beschäftigte er den selbsternannten Baron d'Hancarville, trotz dessen mehr als fragwürdigem Charakter. D'Hancarville hatte schon für Sir William Hamilton den ersten Band von dessen Vasenwerk verfaßt, war, weil heftig verschuldet, mit den Druckplatten nach Florenz durchgebrannt, fand hier kurz eine Anstellung beim Großherzog, doch die Gläubiger holten ihn ein, Sir William konnte ihn auslösen, um nicht nur sein reich illustriertes Vasenwerk zu retten: d'Hancarville war auch mit der Abfassung des Katalogs der übrigen Sammlungen von Sir William beschäftigt, zwei Manuskriptbände sind im British Museum erhalten. ${ }^{10}$

Und spätestens hier finden sich d'Hancarvilles mystische Spekulationen über den Ursprung der Künste ein erstes Mal breit entfaltet. Ihn interessierten besonders antike Gemmen, Siegel in Skarabäusform, Amulettsteine, die er extrem früh datierte und an okkulte Mysterien band, vor allem an den Kult von Bacchus und Ceres in Eleusis und an den Kult der für ihn ältesten Gottheit,

9 Ernst 1992 (wie Anm. 3), 173-184; Kat. Grand Tour (wie Anm. 6), 257-258, Kat. Nr. 215. Ronald Paulson: Emblem and Expression. Meaning in English Art of the Eighteenth Century, London 1975, 138-148, 152-153; Oliver Millar: Zoffany and his Tribuna, London 1966.

10 Zu d'Hancarville: Francis Haskell: Past and Present in Art and Taste. Selected Essays, New Haven/London 1987, 30-45 und Kat. Vases and Volcanoes (wie Anm. 6), 45-51, 96-100; Ernst 1992 (wie Anm. 3), 178-180. 
der Erdgöttin Kybele. Noch weiter zurück zu reichen schienen ihm große formlose, aber geheiligte Steine, die d'Hancarville "pierres parlantes“ oder , animées" nannte, er suchte Rechtfertigung dafür in antikem Schrifttum in den gewagtesten Konstruktionen, immer beeindruckend jedoch in der Mischung aus vielfältigen Quellenverweisen und höchst suggestiver Darstellungsweise. ${ }^{11}$

D'Hancarville stand mit derartigen Spekulationen zeitgenössisch nicht allein. Richard Payne Knight, mit allen bisher genannten Antiquaren und Sammlern in regem Austausch, publizierte 1786 An Account of the Remains of the Worship of Priapus lately existing at Isernia, in the Kingdom of Naples in two letters; one from Sir William Hamilton, K. B. His Majesty's Minister at the Court of Naples, to Sir Joseph Banks, Bart.President of the Royal Society; And the other from a Person residing at Isernia; to which is added, (und das stammt von Richard Payne Knight) $A$ Discourse of the Worship of Priapus, And its Connexion with the mystic Theology of the Ancients. Es wird nicht erwähnt, wer die Publikation finanziert hat: die $S_{0}$ ciety of Dilettanti. Sir William hatte sich fünf der phallischen Exvotos zur Verehrung der Heiligen Cosmas und Damian aus Isernia beschaffen können und sie 1784 bei seinem Besuch in England dem British Museum übergeben, wo sie neben seiner Kollektion antiker Priapi aufgestellt wurden. Sie zieren in versammelter Form das Titelblatt von Richard Payne Knights Traktat. ${ }^{12}$

Was aus alledem erhellt, ist das Folgende, das auch zum Verständnis des Museum Britannicum nicht unwichtig ist: die englische antiquarische Tradition wird vom Adel des 18. und des beginnenden 19. Jahrhunderts getragen, er nutzt sie zur Formulierung einer Privatmythologie, er sucht nach einem Ausgleich zwischen privatem Anspruch und öffentlicher Wirksamkeit, er treibt die Wissenschaft voran, indem er sie instrumentalisiert, er will, so wurde es auch zeitgenössisch formuliert, Kunst und Leben zusammenfallen lassen, die antiken Überbleibsel reanimieren (darum war die Entdeckung des Nachlebens antiker Kulte in christlicher Gegenwart so wichtig), vor allem aber ein Modell zur Erklärung der Ursprünge von Kult, Kunst und Wissenschaft entwickeln, mit entschiedenem Rekurs auf antikes Schriftum. Man sollte diese Form der Geschichtsschreibung nicht gering schätzen, nur weil sie nicht dem kontinentalen, vor allem deutschen historistischen, geschichtsphilosophischen Modell entspricht, das sich als unaufhaltsam erwies, insbesondere in der musealen Konzeption: die Aufstellung der Werke in Kunstmuseen nach Schulen, vor allem aber in streng chronologischer Form waren das Resultat. Diese Form tendiert dazu, den Gegenständen das Leben auszutreiben.

11 Kat. Vases and Volcanoes (wie Anm. 6), 98-100.

12 Kat. The Arrogant Connoisseur. Richard Payne Knight. 1751-1824, hrsg. v. Michael Clarke/Nicholas Penny, Witworth Art Gallery, Manchester 1982; Kat. Vases and Volcanoes (wie Anm. 6), Kat. Nr. 142. 
Jeder private Kunstsammler, ist er ehrlich und sammelt er nach Gusto, hängt nach wie vor dem englischen Modell an, das die Postmoderne wiederentdeckt hat und mit ihm z. B. Sir John Soanes privates Museum in Lincoln's Inn Fields, in dem er wenigstens für sich selbst und seine Sammlung in drei miteinander verbundenen Häusern seine alte Idee für das Museum der Society of Dilettanti verwirklicht hat. Und auch er vermachte sein Museum testamentarisch dem Staat - mit der Auflage, daß nichts verändert werden dürfte, so daß noch heute sein privater Kosmos vor Augen steht, der ebenfalls um Ursprungsgedanken kreist und seinen Ausgang im Kellergeschoß im ägyptischen Sarkophag von Seti I. nimmt, dem wohl bedeutendsten Stück der ganzen unendlichen, bis in Soanes Gegenwart reichenden Sammlung. ${ }^{13}$ Die Sepulkralkammer mit dem Sarkophag bekommt bezeichnenderweise ihr Licht durch das ganze Haus hindurch vom dome - aus dem Dunkel der Vergangenheit ins Licht der Gegenwart, die die Musealisierung der Vergangenheit in lebendiger Weise unternimmt, gestiftet aus subjektiver Sicht. Der Sarkophag war erst 1815 bei von Engländern geleiteten und finanzierten Ausgrabungen in der Nekropole des ägyptischen Theben entdeckt, nach England verschifft und im British Museum deponiert und ihm zum Kauf angeboten worden. Das Museum konnte oder wollte die geforderten 2.000 Pfund nicht aufbringen, so schlug Sir John Soane $1824 \mathrm{zu}$ und kehrte noch einmal den Weg der Kunstwerke um: aus dem Museum in die Privatsammlung, die wieder zum Museum werden sollte. Den Adventus des Sarkophags ließ Soane mit einem dreitägigen Fest feiern. In Soanes eigener Beschreibung zum Sarkophag heißt es, er sei „covered externally and internally with hieroglyphics, comprehending a written language, which is to be hoped the labours of modern literati will render intelligible" ${ }^{14}$ Schon zwei Jahre zuvor hatte Jean-François Champollion das Rätsel der Hieroglyphen mit Hilfe des Steins von Rosette, der sich bekanntlich im British Museum befindet, gelöst und damit einen weiteren Baustein zur Entmythologisierung und Historisierung der Frühgeschichte geliefert. Das Ende der britischen Sammler- und Sammlungskultur zeichnet sich deutlich ab.

Den Anfang vom Ende markiert das Museum Britannicum bzw. Sir Hans Sloanes Stiftung 1744. Die Publikation fußt offenbar gänzlich auf Beispielen aus Sloanes kunst- und wunderkammerartigen Sammlung. Aus einem unglaublichen Wust, zu dem 32.000 Münzen und Medaillen, 2.256 Edelsteine, 5.843 Muscheln, 5.439 Insekten oder 12.506 Pflanzen gehörten, von der 50.000 Bände umfassenden Bibliothek zu schweigen, wählten die Autoren den Bruchteil eines Bruchteils, noch dazu nach heute geradezu irrwitzig erscheinenden Kriterien

13 A New Description of Sir John Soane's Museum, London ${ }^{2} 1969,35-37$.

14 Ebd., 36. 
aus, offenbar durchgehend winzige und möglichst unbedeutende Objekte, die dennoch „curiosity“ wecken sollten.

Wodurch? Durch absolute, geradezu mikroskopische Nähe und absolute Genauigkeit in der Wiedergabe: Porengenauigkeit. Die Technik der Tafeln ist mir zuerst ein Rätsel gewesen. Grundlage ist die Radierung, die Autoren verwahren sich ausdrücklich in der Einleitung gegen die Verwendung von Kreuzschraffuren als einem Kunstmittel, das der Naturerscheinung nicht nahe genug sei. Die Stecher haben sie in seltenen Fällen, kaum sichtbar, dennoch verwendet. Die zarten tonalen Übergänge im Flächenton, der auf die Wiedergabe von Stofflichkeit und Glanz zielt, weisen jedoch ein ungewöhnlich feines Korn auf, das zu diesem Zeitpunkt die in den fünfziger Jahren erfundene Aquatinta liefern konnte. Allerdings muß die Aquatinta zur Tonabstufung jeweils neu ätzen, was feine Übergänge im Grunde genommen verhindert, vielmehr unterschiedliche tonale Flächen eher aneinanderstoßen läßt. Hier jedoch sind die Übergänge so fein, daß es sich wohl eher um eine gänzlich neue, aus Frankreich importierte Technik handeln dürfte: „vernis mou“, Weichgrundätzung, so gut wie gleichzeitig in England von Thomas Gainsborough und George Stubbs verwendet. Die Feinheit ist schier atemberaubend.

Die Autoren, die sich als Maler bezeichnen, argumentieren ausdrücklich gegen jede akademische Idealisierung, haben Joshua Reynolds' permanente Warnung vor der Wiedergabe der „minutiae“, der „particularities“ und „peculiarities" im Visier (vor allem im 4. und 6. Diskurs von 1771 und 1774) und forcieren das genaue Gegenteil. ${ }^{15} \mathrm{Ihr}$ Nachahmungsbegriff diskreditiert die Reynoldssche Auffassung namentlich als manieriert, sieht ihn als einen „Nature-Mender“, der Flickschusterei an der Natur betreibt, bloße Effekthascherei verfehle die Natur, sie wollen „every minute part“ entdecken, fordern $\mathrm{Na}$ turhingabe, die die Frage eines Stils der Darstellung gar nicht erst aufkommen läßt, und auch im Text verzichten sie nachdrücklich auf jedes „embellishment“, „plain writing“ ist das Ziel. ${ }^{16}$ Eine zweite Kunstform neben der akademischen soll etabliert werden, wie sie auch von Künstlern betrieben wurde, die sich (noch) der übermächtigen Akademie verweigerten, wie Joseph Wright of Derby und George Stubbs, die im Konkurrenzunternehmen der Society of Artists ausstellten, bei der auch Stecher Zutritt und Ausstellungsmöglichkeit hatten. Es sind die Künstler, die nicht selten einen naturwissenschaftlichen Anspruch an ihre Kunst stellen, die aus der Provinz stammen und - verkürzt gesagt - der industriellen Revolution, ihrem Erkenntnisstand und Wirklichkeitsverständnis zuarbeiteten.

15 Sir Joshua Reynolds: Discourses on Art, hrsg. v. Robert R. Wark, New Haven/London ${ }^{3} 1988,57-62,69-72,102-103$.

16 Rymsdyck 1791 (wie Anm. 1), V. 
Wright beschäftigte zur Wiedergabe seiner Gemälde professionelle Mezzotinto-Stecher von höchster Qualifikation, die die zartesten tonalen Übergänge zu schaben in der Lage waren. Stubbs wollte seine ungemein detaillierten Zeichnungen zur Anatomie des Pferdes von 1756-58 ebenfalls von Spezialisten stechen lassen - sie lehnten reihenweise aufgrund der Komplexität ab. Stubbs brachte sich das Stechen selbst bei, publizierte The Anatomy of the Horse 1766 im Kupferstich, verfeinerte die Technik im folgenden für die Wiedergabe seiner Werke extrem und erzielte tonale Feinheiten sondergleichen schließlich durch eine hochkomplexe Mischung, bestehend aus Stich und Radierung als Basis und stipple (Punktiermanier), Aquatinta und gelegentlich soft-ground etching (vernis mou, Weichgrundätzung) als Differenzierungsmittel. ${ }^{17}$ In die ohne Newtons Farbtheorie in den Opticks nicht denkbare Geschichte der Verfeinerung des Sehens auf naturwissenschaftlicher Basis gehören auch die Wiedergaben im Museum Britannicum. Zugleich aber scheint die Vorstellung von der Hingabe ans Detail als Erkenntnismedium in physikotheologischer Tradition zu stehen: auch das scheinbar nichtigste und winzigste, das was „perhaps quite indifferent" ist, ist es wert, als Gottes Schöpfung betrachtet und studiert zu werden. Mit Barthold Heinrich Brockes Motto zu seinem „Irdischen Vergnügen in Gott“ von 1721-48 gesprochen: „Nicht der Planeten Gröss’ allein; / Ein Stäubchen, ist bewunderns werth.“ Und das „Stäubchen“ ist nur in einem extrem genauen Wiedergabemodus zu veranschaulichen.

Daß Gedanken vom mythischen Ursprung der Kunst, vom fortlebenden animistischen Geist, der Verbindung zur Gegenwart hält, wie sie d'Hancarville in Townleys Haus endgültig ausformuliert hat, auch für die Autoren des Museum Britannicum eine Rolle gespielt haben, sei anhand eines einzigen Beispiels verfolgt. Es handelt sich um einen auf Tafel XXIII abgebildeten Bildstein (Abb. 2), einen gemusterten Schiefer, zu den sogenannten Grapholithi, den bezeichneten Steinen, gehörig, auf dem die „hand of nature“ eine schöne Landschaft mit Bäumen, Pflanzen und Wolken gemalt hat - ausdrücklich keine klassische, sondern eine Ruisdaelsche nach der Natur. Die Autoren kennen die Tradition derartiger Bildsteine, sie zitieren den Ursprung dieser Tradition, Plinius' Bemerkungen zum Achat des Königs Pyrrhus oder Athanasius Kirchers Bewunderung für derartige „lusus naturae“ in seinem Werk Mundus subterraneus von 1664 , in dem die Rede davon ist, daß religiöse Schriftzeichen sich auf Steinen

17 Zu den Mezzotinti nach Wright: Kat. Wright of Derby, hrsg. v. Judy Egerton, Tate Gallery, London 1990, 231-258; zu Stubbs: Kat. Stubbs and the Horse, hrsg. v. Malcolm Warner/Robin Blake, Kimball Art Museum, Fort Worth; Walters Art Museum, Baltimore; National Gallery, London, New Haven/London 2004, Kap. 2, 19-41; Kat. George Stubbs 1724-1806, Tate Gallery, London 1984, 218-241, bes. 224; George Stubbs: The Anatomy of the Horse, hrsg. v. Constance-Anne Parker, London 2005 (nach der Edition von 1853 mit dem kompletten Text). 


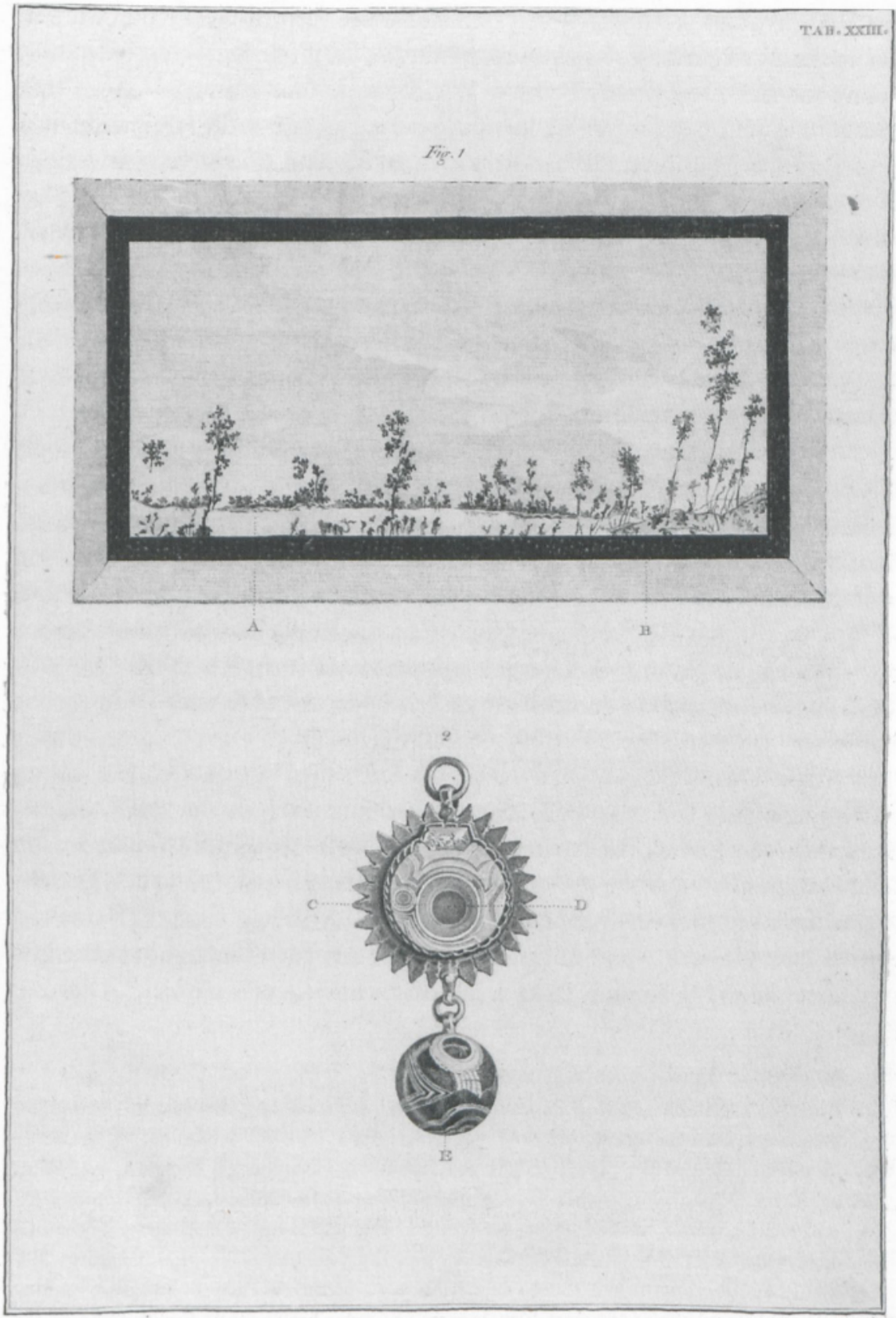

Abb. 2: Museum Britannicum, Tafel XXIII, Fig. 1 Bildstein 
fanden. ${ }^{18}$ Worauf die Autoren verblüffenderweise nicht hinweisen, obwohl es ihnen durchaus geläufig gewesen sein müßte, ist die große Sammlung von Bildsteinen in der Royal Society. ${ }^{19}$ Diese Wunderwerke der Natur, in denen ihre Gestaltungskraft zum Ausdruck kommt, sind auch breit in der Traktatliteratur illustriert worden, ihren Ort fanden sie in kunst- und wunderkammerartigen Sammlungen. Conrad Gesner (1565) sah in ihnen eine eigene Klasse der Fossilien, Ulisse Aldrovandi (1648) war besonders auf Tierdarstellungen im Marmorschnitt aus, Robert Plot (1677) liebte Stern- und Muschelsteine. ${ }^{20} \mathrm{Daß}$ auf den Bildsteinen Natur Kunst täuschend nachahmt, konnte als Rechtfertigung eines antiidealistischen Nachahmungsbegriffes gelesen werden, wie ihn die Autoren des Museum mit Aplomb vertraten. Natur ist, wie sie ist, nicht, wie sie sein soll. Natur ist die Kunst Gottes.

Nun verschränken sich bei der Beurteilung der Bildsteine verschiedene Denktraditionen zum Verhältnis von Natur und Kunst, von denen zumindest zwei sorgfältig geschieden werden sollten. Für beide hat Plinius Grund gelegt. Es geht um Notwendigkeit oder Zufall. Der Achat des Königs Pyrrhus soll Apoll und die neun Musen gezeigt haben. Sie waren, heißt es ausdrücklich bei Plinius, nicht von Künstlerhand geschaffen, sondern „naturae sponte“, nach dem Willen der Natur. ${ }^{21}$ Das Adverb „sponte“ kann nicht nur „nach dem Willen“ bedeuten, sondern genauerhin auch ,aus eigenem Antrieb“ oder "ohne Hilfe von anderer Seite“. Die Natur und nur sie ist in eigener Absicht tätig geworden. Dieses Beispiel findet sich im Buch über die Steine. Die Gründungsgeschichte der anderen Denktradition findet sich im 35. Buch, das bekanntlich den Farben der Malerei und der Plastik gewidmet ist, und betrifft den Künstler Protogenes, der verzweifelt den Schaum am Mund eines Hundes zu malen suchte, in verschiedenen Anläufen nicht zurecht kam, die Farbe immer wieder abwischte und schließlich in Wut den Farbschwamm auf das Bild warf, worauf der Schaum perfekt dargestellt schien: „casus pinxerit“ - der Zu-

18 Rymsdyck 1791 (wie Anm. 1), 59-60.

19 Lorraine Daston/Katharine Park: Wunder und die Ordnung der Natur. 1150-1750, Berlin 2002 (zuerst engl. 1998), 352.

20 Horst W. Janson: The „Image Made by Chance“ in Renaissance Thought, in: Millard Meiss (Hrsg.), De artibus opuscula XL. Essays in Honor of Erwin Panofsky, New York 1961, Bd. 1, 254-266; ders., Chance Images, in: Philip P. Weiner (Hrsg.), Dictionnary of the History of Ideas. Studies of Selected Pivotal Ideas, Bd. 1, New York 1973, 340-353; Jurgis Baltrusaitis: Imaginäre Realitäten, Fiktion und Illusion als produktive Kraft. Tierphysiognomie - Bilder im Stein - Waldarchitektur - Illusionsgärten, Köln 1984 (zuerst franz. 1983), 55-89; Horst Bredekamp: Antikensehnsucht und Maschinenglauben. Die Geschichte der Kunstkammer und die Zukunft der Kunstgeschichte, Berlin 1993, 20, 42, 64-65 (überarbeitete Neuausgabe Berlin 2000, 19 ff., 42-43, 66 ff.); Daston/Park 2002 (wie Anm. 19), 327-340.

21 C. Plinius Secundus d. Ä.: Naturkunde, Lateinisch-deutsch, Buch 37: Steine. Edelsteine, Gemmen, Bernstein, hrsg. u. übers. v. Roderich König in Zusammenarbeit mit Joachim Hopp, München 1994, 18-19 (III, 5 u. 6). 
fall hat gemalt; und gleich darauf heißt es noch einmal „fecitque in pictura fortuna naturam“, „so hat in der Malerei der Zufall die Naturwahrheit geschaffen “. ${ }^{22} \mathrm{Da}$ in der Traktatliteratur häufig in Bezug auf die Steinbildungen vom Spiel der Natur die Rede ist, hat die Forschung nicht selten beide Traditionen anstandslos vermischt. Dabei entgeht ihr der tiefere Sinn der Steinbildungen selbst. Zumal sich die Protogenes-Geschichte leicht mit Leonardos Bemerkungen zur Möglichkeit, auf fleckigen Mauern Landschaften, ja, ganze Schlachten zu erkennen, verbinden ließ ${ }^{23}$ Leonardo, das sei betont, hebt nicht auf die Zufallsbildungen ab, sondern auf die Projektionsleistung und das Vorstellungsvermögen des Betrachters, der das Gesehene und Imaginierte als Anregung nutzen und dann in eine vollständige und klare Form bringen kann. Schon Horst W. Janson hat nachweisen können, daß das Leonardosche Verfahren bei Alberti in De Statua vorgebildet ist, aber auch antike Vorläufer besitzt. ${ }^{24}$

Dieses Verfahren der künstlerischen Konkretisierung des im Naturbild Imaginierten verband sich, vor allem im 17. Jahrhundert, insofern mit den Bildsteinen, als deren zumeist Landschaften ähnelnde Strukturen weitergemalt wurden, indem Figuren inseriert wurden, die sich mit dem von Natur aus Angelegten vertrugen. Philipp Hainhofer in Augsburg trieb einen schwunghaften Handel mit derartigen fortgemalten Steinen als Kunst- und Wunderkammerobjekten. Florentiner Steine, so schrieb er, seien besonders geeignet dafür. ${ }^{25}$ Der Florentiner Stein wird zu einem terminus technicus, der auch noch dem Museum geläufig ist. Wichtiger in unserem Zusammenhang ist jedoch die Tradition, in der die Natur allein, selbstbildend tätig wird. Denn ihr läßt sich die Kirchersche Kosmogonie-Vorstellung von einer unterirdisch-mineralogischen Welt verbinden, aus der die Kette der Wesen von Gott bewirkt auftaucht, in der die Steine als Organismen verstanden werden, aus denen sich in alchemistischer Metamorphose die Pflanzen und Lebewesen bis hin zum Menschen entwi-

22 Ders., Buch 35: Farben, Malerei, Plastik, hrsg. u. übers. v. Roderich König in Zusammenarbeit mit Gerhard Winkler, München 1978, 78-81, (1. Zitat 78; 2. Zitat 80)

23 Zum Begriff „lusus naturae“: Jean Céard: La nature et les prodigues: L'insolite au XVIe siècle en France, Genf 1977; Baltrusaitis 1984 (wie Anm. 20), 59-60; Paula Findlen: Jokes of Nature and Jokes of Knowledge. Playfulness of Scientific Discourse in Early Modern Europe, in: Renaissance Quarterly 43, 1990, 292-331; Bredekamp 1993/2000 (wie Anm. 20), 66 ff., 77 u. Anm. 139; zu Leonardos Fleckenlandschaften ist die Literatur Legende; für den hiesigen Zusammenhang die Quellen: Leonardo da Vinci. Sämtliche Gemälde und die Schriften zur Malerei, hrsg. von André Chastel, München 1990, 212, 385; die Tradition des Topos: Ernst Kris/Otto Kurz: Die Legende vom Künstler. Ein geschichtlicher Versuch. Mit einem Vorwort von Ernst H. Gombrich, Frankfurt a. M. 1980 (zuerst 1934), 71-73; Janson 1961 (wie Anm. 20), 260-262, 264-265; Baltrusaitis 1984 (wie Anm. 20), 55.

24 Janson 1961 (wie Anm. 20), 254-255, 260-261.

25 Baltrusaitis 1984 (wie Anm. 20), 55-59; Daston/Park 2002 (wie Anm. 19), 301-305, 312, $327-328$. 
ckeln. ${ }^{26}$ In dieser bis Gottfried Wilhelm Leibniz oder Jean-Baptiste-René Robinet wirksamen Vorstellung sind die Bildsteine als Präfigurationen der belebten Welt zu verstehen. Ausdrücklich heißt es bei Robinet: „Es ist kein Spiel des Zufalls, sondern es sind Erzeugnisse einer Vielzahl von besonderen Verwirklichungsweisen des einzigen Urbildes aller Wesen [...]“.27 Wenn im 1777 in Nürnberg publizierten Recueil von Georg Wolfgang Knorr und Johann Ernst Immanuel Walch dagegen mit Bezug auf die Bildsteine von zufälligen Bildungen und Spielen der Natur die Rede ist ${ }^{28}$, dann wird das Spiel mit Ähnlichkeiten als bedeutungslos verstanden, und die Kette der Wesen ist zerrissen, Entmythologisierungs- und Säkularisierungsvorstellungen greifen Raum. Dieser Punkt ist im Museum noch nicht erreicht. Der Antihistorismus des Adels, der mit antiquarisch fundierten Privatmythen die Tradition des Allzusammenhanges am Leben hält, steht davor.

Abschließend sei vorsichtig ein Gedanke geäußert, der angeregt ist von der Vorstellung, daß die Natur als Präfiguration der Kunst zu denken ist. In den Kunst- und Wunderkammern finden sich Objekte, die den Übergang von unterirdischer Natur zu oberirdischer Kunst ausdrücklich thematisieren. Ernst Kris, der diesen Typus untersucht hat, schildert den Entwurf eines Tischbrunnens als Tafelaufsatz von Wenzel Jamnitzer folgendermaßen: „Auf mächtigen Steinblöcken ruht der ganze Brunnen. Man ist versucht, ihm eine inhaltliche Motivierung zu unterschieben, etwa den Gedanken, daß sich auf dem sicheren Boden der Natur die reichentfaltete Pracht des Kunstwerkes aufbaue“". ${ }^{29}$ In der Tat ist der bloße Fels als Fuß des Aufsatzes, als Grotte, als Revier der aus dem Untergrund kommenden Zwischenwesen, der Wassernymphen, markiert. Bei einem 1596 datierten Kugellaufautomaten von Christian Markgraf findet im unteren Teil reales Felsgestein Verwendung, die darauf aufwachsenden Grottenbögen dagegen sind gemalt. ${ }^{30}$ Es ist die Grotte der Diana, die mit ihren

26 Arthur O. Lovejoy: Die große Kette der Wesen. Geschichte eines Gedanken, Frankfurt a. M. 1985 (zuerst engl. 1933), insbes. zur Leibnizschen Tradition, 176-220; zu Kircher bes.: Baltrusaitis 1984 (wie Anm. 20), 64-71; Bredekamp 2000 (wie Anm. 20), Kap. Die historische Kette, 19-39.

27 Jean-Baptiste-René Robinet: De la nature, Bd. 4, Amsterdam 1766, 212, zit. bei Baltrusaitis 1984 (wie Anm. 20), 81.

28 Georg Wolfgang Knorr/Johann Ernst Immanuel Walch: Recueil des monuments des catastrophes que le globe terrestre a essuyées, Nürnberg 1777, zit. bei Baltrusaitis 1984 (wie Anm. 20), 82. Zu den Historisierungskonsequenzen s. auch: Wolf Lepenies: Das Ende der Naturgeschichte. Wandel kultureller Selbstverständlichkeiten in den Wissenschaften des 18. und 19. Jahrhunderts, Frankfurt a. M. 1976; Ernst 1992 (wie Anm. 3), Kap. VII, 201-227.

29 Ernst Kris: Der Stil „rustique“. Die Verwendung des Naturabgusses bei Wenzel Jamnitzer und Bernard Palissy, in: Jahrbuch der Kunsthistorischen Sammlungen in Wien NF 1, 1926, 137-208, Zitat 151.

30 Ebd., 171-172. 
Nymphen von Aktäon überrascht wird. Und in Ovids Metamorphosen, in denen die Geschichte des unglücklichen Jägers erzählt wird, findet eben dieses $\mathrm{Na}$ tur-Kunst-Verhältnis seine zentrale Formulierung: „Hier lag“, heißt es zu Dianas Grotte, „eine umschattete Höhle im hintersten Winkel, / Keinerlei Schöpfung der Kunst; die Natur, in eigener Erfindung, / Hatte ein Kunstwerk geformt: aus lebendigem Bimsstein und leichtem Tuff einen Bogen gezogen, der hier an der Stelle gewachsen “. ${ }^{31}$ Bei Ovid bleibt die Natur die Künstlerin, Mārkgrafs Automat umspielt den Übergang von Natur zu Kunst.

Es ist die Geburt von Kunst und Gegenstand aus dem Schoß der Mutter Erde, architekturterminologisch verkörpert von der Rustica. Vasari läßt die Grotte, die Giovanni da Udine im Garten der Villa Madama anlegte, ,aussehen, als sei sie wahrhaftig ein natürlicher Gegenstand “. ${ }^{32}$ Dieses Umspielen der Grenze hat eine lange Tradition, bis hin zur romantischen Arabeske eines Philipp Otto Runge oder Eugen Napoleon Neureuther. Die Arabeske wächst aus dem abstrakten ornamentalen Urgrund in die gegenständliche Konkretion auf. Auch in der romantischen Arabeske gibt es einen Quellpunkt, einen, wie Friedrich Schlegel schreibt und Walter Benjamin zitiert, „Übergang, der immer ein Sprung sein muß": im romantischen Denken leistet diesen Sprung die Reflexion. ${ }^{33}$ Auch die Leonardosche Projektion, die die flächige Mauer in der Vorstellung zu einer Landschaft macht, braucht den Sprung der Imagination. Daß dies am Anfang des 16. Jahrhunderts vorgetragen wird, von einem Künstler, der wie kein anderer die Natur untersucht und sich auf sie verpflichtet, der zeichnerisch in ungezählten Anläufen das Entstehen der Naturformen und abläufen umkreist und im sfumato nach einem tonalen Äquivalent für atmosphärische Übergänge sucht, läßt den Gedanken aufkommen, ob nicht zeichnerische und malerische Skizze, deren Eigenwert besonders in der venezianischen Kunst des 16. Jahrhunderts entdeckt wurde ${ }^{34}$, dem geschilderten Naturbegriff

31 Publius Ovidius Naso: Metamorphosen, hrsg. u. übers. v. Hermann Breitenbach, Stuttgart 1988 (zuerst 1958), 3. Buch, 95, Z. 156-160.

32 Giorgio Vasari, Le Vite, hrsg. v. Gaetano Milanesi, Bd. 6, Florenz 1881, 556; Giorgio Vasari, Le Vite, hrsg. v. Rosanna Bettarini u. komm. v. Paolo Barocchi, Bd. 5, Florenz 1984, 451. - Zur Erde als Mutterleib: Horst Bredekamp: Die Erde als Lebewesen, in: kritische berichte $9,1981,5-37$.

33 Friedrich Schlegel: 1794-1802. Seine prosaischen Jugendschriften, hrsg. v. Jakob Minor, Wien 1906, Bd. 2, 176; Walter Benjamin: Der Begriff der Kunstkritik in der deutschen Romantik, hrsg. v. Hermann Schweppenhäuser, Frankfurt a. M. 1972, 22; Karl Konrad Polheim: Die Arabeske. Ansichten und Ideen aus Friedrich Schlegels Poetik, München/Paderborn/ Wien 1966; Werner Busch: Die notwendige Arabeske. Wirklichkeitsaneignung und Stilisierung in der deutschen Kunst des 19. Jahrhunderts, Berlin 1985, 44-75; Günter Oesterle: Stichwort „Arabeske“, in: Ästhetische Grundbegriffe. Historisches Wörterbuch in sieben Bänden, Karlheinz Barck u. a. ( Hrsg.), Bd. 1, Stuttgart 2000, 272-286.

34 Siehe bes.: Beiträge zur Geschichte der Ölskizze vom 16. bis zum 18. Jahrhundert. Ein Symposion aus Anlaß der Ausstellung „Malerei aus erster Hand - Ölskizzen von Tintoretto 
das Entscheidende zur Rechtfertigung ihres Wirklichkeitszugriffs verdanken. Denn Zeichnung und Ölskizze in dieser Tradition zielen nicht auf die Festlegung des Gezeigten im Umriß, zielen nicht auf Objektivierung und idealistische Normierung, sondern deuten bloß an, suchen im Prozeß des Malens und Zeichnens nach der Form, belassen die Dinge im Vorläufigen und brauchen so den Anteil, die Projektion des Betrachters. Sie zeigen das Entstehen der Dinge aus dem abstrakten Stoff der Kunstmittel in die künstlerische Konkretion. Die klassisch-akademische Kritik hat diese Form der Kunst immer auf einen bloBen Naturzugriff reduziert. Aber vielleicht ist es gerade diese Form bis hin zur extremen Wirklichkeitsverpflichtung des Museum Britannicum, die die Kunst in durchaus naturmystischer Tradition als Verlebendigung begreift.

bis Goya“ im Herzog Anton Ulrich-Museum Braunschweig, hrsg. v. Herzog Anton UlrichMuseum Braunschweig, Braunschweig 1984, bes. der Beitrag von Linda Freeman Bauer: Some Early Views and Uses of the Painted Sketch, 14-24. 